\title{
Conditional Quantile Polynomial Distributed Lag Model with an Application to Rubber Price Returns
}

\author{
Kwadwo Agyei Nyantakyi \\ Ghana Institute of Management and Public Administration (GIMPA), Greenhill College, Business School, Box AH 50, Achimota \\ Accra, Ghana
}

\begin{abstract}
Impacts of returns on assets are not instantaneously felt, since there is lag period. In this paper we consider the problem of developing a model for the conditional QPDL (quantile polynomial distributed lag) and investigate the influences of the conditioning variables on the location, scale and shape parameters of the QPDL model. As an economic application, we consider the production of rubber and its price returns of Sri Lanka. From the analysis we observed that the QPDL model applications were better estimators than the PDL (Polynomial Distributed Lag) models.
\end{abstract}

Key words: Asset returns, percentiles, parameter estimators, production.

\section{Introduction}

Impacts of returns on assets are not instantaneous, due to the lag effect. Some of agricultural prices have some evidences of lag behavior [1-3]. Various economic models such as geometric distributed lag model [4-6], adaptive expectations and partial adjustment models [7], rational expectation model [8], polynomial distributed lag model $[9,10]$. have being used for modelling these lags. Generally the conditional mean, used in theses estimations, provides only single summary measure for the conditional distribution of response (predictand) - the mean - given the predictor whereas quantile regression provides a more complete picture of the conditional distribution, thus, there is variation across the quantiles which exhibit different behavior [11, 12]. Also using conditional means for modelling price returns are not accurate since they present evidence of symmetric and cyclic responses [13, 14]. In the light of these, estimation of the QAR (QuantileAutoregressions) which focuses on the evolution of conditional quantiles in time instead of conditional means as the usual AR

Corresponding author: Kwadwo Agyei Nyantakyi, Ph.D., research fields: time series, quantile regression and econometrics/economics. E-mail:knyantakyi@yahoo.com. (autoregression) becomes very important [15]. Quantile estimates in modelling are very important because it models the relation between a set of predictor variables and specific percentiles (or quantiles) of the response variable [16]. In order to address some of the issues raised above, Koenker and Ziao [17] developed a method for the Estimation of QAR models. We therefore in this research propose a conditional QPDL (quantile polynomial distributed lag) model to study the structural changes in the time series model by estimating the parameters of the QPDL model. As an economic application, we consider Sri Lanka rubber production and its price returns.

The objectives of this study are to develop a new method for the estimation of distributed lags using quantiles, to study the effects of rubber production on its price returns, and to compare the QPDL model and the PDL model

\section{Materials and Methods}

\subsection{Data Source}

In order to answer the issues raised above, secondary annual data was collected from FAOSTAT, food balance sheet, price statistics, available with the 
Department of Census and Statistic Sri Lanka [18], and the World Bank (pink sheet) [19]. These data comprise of the production, imports, exports and prices of rubber. The rubber data ranges from 1961-2011.

\subsection{Statistical Software}

The R software, with the package "Quantreg” was used in fitting the conditional quantile polynomial distributed lag models.

\subsection{The Model and Assumption}

Given the autoregressive distributed lag

$$
\begin{aligned}
y_{t}=\alpha & +\beta_{0} X_{t}+\beta_{1} X_{t-1}+\beta_{2} X_{t-2}+\beta_{3} X_{t-3}+ \\
& ---+\beta_{n} X_{t-k}+\varepsilon_{t}
\end{aligned}
$$

With $y_{t}$ dependent variable and $X_{t}$ independent variable and $\left\{\varepsilon_{t}, 1 \leq t \leq k\right\}$ are independent identically distributed random errors.

$$
\begin{gathered}
X_{t-1}=X_{t-2},---, X_{t-k}, \\
F_{t-1}=\sigma\left\{X_{u}, u \leq t-1\right\} \quad \text { the } \sigma-\text { algebra }
\end{gathered}
$$
generated by the observations up to time $t-1$.

The polynomial distributed lag by Almon [9] can be written as

$$
y_{t}=\varphi+\sum_{i=0}^{k} \beta_{i} X_{t-i}+\varepsilon_{t}
$$

With $k$ number of lags, and the $\beta_{i}{ }^{\prime} s$ can be approximated by suitable polynomials. That is

$$
\beta_{i}=a_{0}+a_{1} i+a_{2} i^{2}+a_{3} i^{3}+---+a_{n} i^{n}
$$

Thus, for the $\mathrm{n}^{\text {th }}$ degree polynomial with $k$ number of lags we have

$$
\begin{array}{r}
y_{t}=\varphi+\sum_{i=0}^{k}\left(a_{0}+a_{1} i+a_{2} i^{2}+a_{3} i^{3}+---\right. \\
\left.+a_{n} i^{n}\right) X_{t-i}+\varepsilon_{t} \\
y_{t}=\varphi+\sum_{i=0}^{k} a_{0} X_{t-i}+\sum_{i=0}^{k} a_{1}(i) X_{t-i}+ \\
\sum_{i=0}^{k} a_{2}\left(i^{2}\right) X_{t-i}+\sum_{i=0}^{k} a_{2}\left(i^{2}\right) X_{t-i}+ \\
\sum_{i=0}^{k} a_{3}\left(i^{3}\right) X_{t-i} \pm--+\sum_{i=0}^{k} a_{n}\left(i^{n}\right) X_{t-i}+\varepsilon_{t}
\end{array}
$$

as defined by Chen, et al [20], Koenker and Bassett [21]. Given a uniformly standard random variable identically independently distributed sequence of random variables $\left\{\psi_{t}\right\}$, we define the new model as conditional QPDL for the $\mathrm{n}^{\text {th }}$ degree polynomial as given below:

$$
\begin{gathered}
y_{t}=\varphi\left(\psi_{t}\right)+\sum_{i=0}^{k} a_{0} \psi_{t} X_{t-i}+\sum_{i=0}^{k} a_{1} \psi_{t}(i) X_{t-i} \\
+\sum_{i=0}^{k} a_{2} \psi_{t}\left(i^{2}\right) X_{t-i}+ \\
\sum_{i=0}^{k} a_{2} \psi_{t}\left(i^{2}\right) X_{t-i}+\sum_{i=0}^{k} a_{3} \psi_{t}\left(i^{3}\right) X_{t-i}+-- \\
-+\sum_{i=0}^{k} a_{n} \psi_{t}\left(i^{n}\right) X_{t-i}+\varepsilon_{t}
\end{gathered}
$$

Assuming $\left\{\varepsilon_{t}, 1 \leq t \leq n\right\}$ are independent identically distributed random errors. We can write the lag model as:

$$
\begin{gathered}
y_{t}=\varphi\left(\psi_{t}\right)+a_{0} \psi_{t} \sum_{i=0}^{k} X_{t-i}+a_{1} \psi_{t} \sum_{i=0}^{k}(i) X_{t-i} \\
+a_{2} \psi_{t} \sum_{i=0}^{k}\left(i^{2}\right) X_{t-i}+
\end{gathered}
$$

$$
\begin{gathered}
a_{2} \psi_{t} \sum_{i=0}^{k}\left(i^{2}\right) X_{t-i}+a_{3} \psi_{t} \sum_{i=0}^{k}\left(i^{3}\right) X_{t-i}+-- \\
-+a_{n} \psi_{t} \sum_{i=0}^{k}\left(i^{n}\right) X_{t-i}+\varepsilon_{t}
\end{gathered}
$$

letting

$$
\begin{gathered}
Z_{0 t}=\sum_{i=0}^{k} X_{t-i} \\
Z_{1 t}=\sum_{i=0}^{k}(i) X_{t-i} \\
Z_{2 t}=\sum_{i=0}^{k}\left(i^{2}\right) X_{t-i} \\
Z_{3 t}=\sum_{i=0}^{k}\left(i^{3}\right) X_{t-i} \\
Z_{n t}=\sum_{i=0}^{k}\left(i^{n}\right) X_{t-i}
\end{gathered}
$$

Then the QPDL can be simplified as:

$$
y_{t}=\varphi\left(\psi_{t}\right)+a_{0}\left(\psi_{t}\right) Z_{0 t}+a_{1}\left(\psi_{t}\right) Z_{1 t}+
$$

$a_{2}\left(\psi_{t}\right) Z_{2 t}+a_{3}\left(\psi_{t}\right) Z_{3 t}+--+a_{n}\left(\psi_{t}\right) Z_{n t}+\varepsilon_{t}$ (6)

The conditional $\alpha$-quantile function (QPDL) can be written as

$$
\begin{gathered}
Q_{y_{t}}\left(\alpha \mid Z_{0 t},---, Z_{n t}\right)=\varphi(\alpha)+a_{0}(\alpha) Z_{0 t}+ \\
a_{1}(\alpha) Z_{1 t}+a_{2}(\alpha) Z_{2 t}+a_{3}(\alpha) Z_{3 t}+---+ \\
a_{n}(\alpha) Z_{n t}+Q_{y t}\left(\varepsilon_{t}\right)
\end{gathered}
$$

where $Q_{y t}\left(\varepsilon_{t}\right)=u_{t}$

This can simply be written as 
where

$$
Q_{y_{t}}(\alpha \mid \xi)=Z_{i t}^{T} a(\alpha)
$$

$$
z_{i t}^{T}=\left(1, Z_{0 t},---, Z_{n t}\right)^{T}
$$

Considering a second degree polynomial, we have

$$
\begin{gathered}
Q_{y_{t}}\left(\alpha \mid Z_{i t}\right)=\varphi(\alpha)+a_{0}(\alpha) Z_{0 t}+a_{1}(\alpha) Z_{1 t}+ \\
a_{2}(\alpha) Z_{2 t}+Q_{y t}\left(\varepsilon_{t}\right)
\end{gathered}
$$

The conditional cumulative probabilities of $\left(Y_{t}\right)$. This is given by

$$
\operatorname{Pr}\left(Y_{t} \leq q\left(Z_{i t}\right) \mid Z_{i t}=z\right)=\alpha \text {. }
$$

We solve the minimization problem

$$
\begin{aligned}
E\left(\left|Y_{t}-q\left(Z_{i t}\right)\right|_{\alpha} \mid Z_{i t}=z\right) & \\
& =\min _{f \in L^{\prime}(u)} E\left(\left|Y_{t}-f\left(Z_{i t}\right)\right|_{\alpha} \mid Z_{i t}=z\right)
\end{aligned}
$$

For a QPDL (2) we have

$$
Y_{t}=\varphi(\alpha)+a_{0}(\alpha) Z_{0 t}+a_{1}(\alpha) Z_{1 t}+a_{2}(\alpha) Z_{2 t}+
$$

Therefore, to solve the minimization problem for the estimates for $\varphi, a_{0}, a_{1}$ and $a_{2}$ we have

$$
\begin{gathered}
S\left(\varphi, a_{0}, a_{1} a_{2}\right)= \\
\sum_{t \mid y_{t} \geq a Z_{i t}}^{k} \alpha\left(\left|Y_{t}-\varphi+a_{0} Z_{0 t}+a_{1} Z_{1 t}+a_{2} Z_{2 t}\right|\right)+ \\
\sum_{t \mid y_{t}<a Z_{i t}}^{k}(1-\alpha)\left(\left|Y_{t}-\varphi+a_{0} Z_{0 t}+a_{1} Z_{1 t}+a_{2} Z_{2 t}\right|\right) \\
=\sum_{t \mid y_{t} \geq a Z_{i t}}^{k} \alpha\left(\left|Y_{t}-Z_{i t}^{T} \omega\right|\right)+\sum_{t \mid y_{t}<a Z_{i t}}^{k}(1- \\
\alpha)\left(\left|Y_{t}-Z_{i t}^{T} \omega\right|\right)
\end{gathered}
$$

where $\omega=\left(\varphi(\alpha), a_{0}(\alpha), a_{1}(\alpha), a_{2}(\alpha)\right)$ denotes the coefficients. $\sum_{t}^{k} \alpha\left|\epsilon_{t}\right|$ and $\sum_{t}^{k}(1-\alpha)\left|\epsilon_{t}\right|$ is called the asymmetric penalty for under prediction and over prediction, respectively.

\section{Results and Discussion}

\subsection{Rubber Production}

The summary statistics of rubber production (in tonne) and returns on rubber between 1964-2011are given in Table 1.

From Table 1 we observe that the average price of rubber between 1964-2011 was USD 1.7029 per year with a minimum price of USD 0.75 and maximum of USD 4.43, respectively. Also it could be seen that the average production of rubber between 1964-2011 was 124,831 tonne with a minimum production of 86,230 and maximum of 159,158 tonne.

The polynomial distributed lag (3-lags) parameter estimates with Proz0 the first polynomial transformation (rubber production lag one), Proz1 the second polynomial transformation and Proz2 the third polynomial transformation, the standard errors and confidence intervals are given in Table 2.

From Table 2, we observed that only the parameter estimates of the second and the third lags are significant and the rest of the estimates are not significant. The R-squared value is 0.52 , that is $52 \%$ of the dependant variable can be explained by the independent variables with an $\mathrm{AIC}=75.79$ and $\mathrm{BIC}=$ 85.148.

We therefore proceed with the QPDL model estimation.

Table 1 Summary statistics for rubber production (in tonne) and price of rubber (USD).

\begin{tabular}{llllll}
\hline Variable & Observations & Mean & Std. Dev & Min & Max \\
\hline Rubber price & 48 & 1.702917 & 0.7033203 & 0.75 & 4.43 \\
Rubber production & 48 & 124,831 & $21,735.91$ & 86,230 & 159,158 \\
\hline
\end{tabular}

Table 2 Polynomial distributed lag regression parameter estimates, standard errors and confidence intervals for production and price of rubber.

\begin{tabular}{llllll}
\hline Price & Coef. & Std. Err. & $\mathrm{t}$ & $\mathrm{P}>|\mathrm{t}|$ & [95\% Conf. Interval] \\
\hline Proz0 & -0.0000137 & $8.34 \mathrm{e}-06$ & -1.64 & 0.108 & -0.00003050 .0000031 \\
Proz1 & 0.000057 & $9.73 \mathrm{e}-06$ & 5.86 & 0.000 & 0.00003740 .0000766 \\
Proz2 & -0.0000198 & $3.24 \mathrm{e}-06$ & -6.11 & 0.000 & $-0.0000263-0.0000132$ \\
Constant & 0.3184857 & 0.4604236 & 0.69 & 0.493 & -0.60943711 .246408 \\
\hline
\end{tabular}

R-squared $=0.52$, RMSE $=0.5015$, Obs $=48$, Prob $>$ F $=0.001$, AIC $=75.79$, BIC $=85.148$. 
The conditional QPDL with 3-lags, regression parameter estimates with Proz0 the first polynomial transformation for rubber production, Proz1 the second polynomial transformation and Proz2 the third polynomial transformation, the standard errors and confidence intervals are given in Table 3, 4,5 and 6 below.

From Table 3, the results show that all parameter estimates are significant except Proz0 and the constant with an R-square of 0.67 .

The resulting equation from Table 3 is:

$$
\begin{aligned}
& \hat{Y}_{t}=0.167703-0.00000459 Z_{0 t}+ \\
& 0.0000349 Z_{1 t}-0.000013 Z_{2 t}
\end{aligned}
$$

Estimating the coefficients of the original variables, we have

$$
\begin{gathered}
\hat{\beta}_{0}=\hat{a}_{0}=-4.59 \mathrm{e}-06 \\
\hat{\beta}_{1}=\hat{a}_{0}+\hat{a}_{1}+\hat{a}_{2} \\
=-4.59 \mathrm{e}-06+0.000035 \\
-0.000013=0.00001731
\end{gathered}
$$

$$
\begin{aligned}
\hat{\beta}_{2}=\hat{a}_{0}+2 \hat{a}_{1}+4 \hat{a}_{2} & \\
& =-4.59 \mathrm{e}-06+(2 * 0.000035) \\
& -(4 * 0.000013)=0.0000132 \\
\hat{\beta}_{3}=\hat{a}_{0}+3 \hat{a}_{1}+ & 9 \hat{a}_{2} \\
& =-4.59 \mathrm{e}-06+(3 * 0.000035) \\
& -(9 * 0.000013)=-0.0000169
\end{aligned}
$$

Therefore, we have the QPDL estimated model as:

$$
\begin{array}{r}
\hat{Y}_{t}= \\
0.1677-0.00000459 X_{0}+0.0000173 X_{t-1}+ \\
0.0000132 X_{t-2}-0.0000169 X_{t-3}
\end{array}
$$

The resulting equation from Table 4 is:

$$
\begin{gathered}
Y_{t}=1260894-0.0000147 Z_{0 t}+0.0000626 Z_{1 t} \\
-0.0000218 Z_{2 t}
\end{gathered}
$$

Although at the 50 percentile from Table 4, all the parameter estimates of the $1^{\text {st }}, 2^{\text {nd }}$, and the $3^{\text {rd }}$ lags are significant except the constant with R-square of $84 \%$ and AIC $=66.28824$. Hence we proceeded to Table 5,

The resulting equation from Table 5 is:

Table 3 Conditional quantile polynomial distributed lag (QPDL) 0.25 quantile regression parameter estimates, standard errors and confidence intervals for production and price of rubber.

\begin{tabular}{lllllll}
\hline Quantile & Price & Coef & Std. Err. & $\mathrm{t}$ & $\mathrm{P}>|\mathrm{t}|$ & [95\% Conf. Interval] \\
\hline 0.25 & Proz0 & $-4.59 \mathrm{e}-06$ & $4.51 \mathrm{e}-06$ & -1.02 & 0.315 & -0.00001370 .0000045 \\
& Proz1 & 0.0000349 & $5.96 \mathrm{e}-06$ & 5.85 & 0.000 & 0.00002290 .0000469 \\
& Proz2 & -0.000013 & $1.74 \mathrm{e}-06$ & -7.46 & 0.000 & $-0.0000165-0.0000095$ \\
& Constant & 0.1677703 & 0.2572442 & 0.65 & 0.518 & -0.35067130 .6862118 \\
\hline
\end{tabular}

Pseudo R-squared $=0.67$, Number of obs $=48$, Prob $>F=0.000$, AIC $=54.81562$.

Table 4 Conditional quantile polynomial distributed lag (QPDL) 0.50 quantile regression parameter estimates, standard errors and confidence intervals for production and price of rubber.

\begin{tabular}{lllllll}
\hline Quantile & Price & Coef & Std. Err. & $\mathrm{t}$ & $\mathrm{P}>|\mathrm{t}|$ & [95\% Conf. Interval] \\
\hline 0.50 & Proz0 & -0.0000147 & $5.42 \mathrm{e}-06$ & -2.71 & 0.009 & $-.0000256-3.78 \mathrm{e}-06$ \\
& Proz1 & 0.0000626 & $6.32 \mathrm{e}-06$ & 9.91 & 0.000 & 0.00004990 .0000754 \\
& Proz2 & -0.0000218 & $2.11 \mathrm{e}-06$ & -10.34 & 0.000 & $-0.0000261-0.0000176$ \\
& Constant & 0.1260894 & 0.2857902 & 0.44 & 0.661 & -0.4498830 .7020617 \\
\hline
\end{tabular}

Pseudo R-square $=0.84$, Number of obs $=48$, Prob $>F=0.000$, AIC $=66.28824$.

Table 5 Conditional quantile polynomial distributed lag (QPDL) 0.75 quantile regression parameter estimates, standard errors and confidence intervals for production and price of rubber.

\begin{tabular}{lllllll}
\hline Quantile & Price & Coef & Std. Err. & $\mathrm{t}$ & $\mathrm{P}>|\mathrm{t}|$ & [95\% Conf. Interval] \\
\hline 0.75 & Proz0 & -0.0000259 & 0.0000105 & -2.48 & 0.017 & $-0.000047-4.84 \mathrm{e}-06$ \\
& Proz1 & 0.0000731 & 0.0000157 & 4.66 & 0.000 & 0.00004150 .0001047 \\
& Proz2 & -0.0000225 & $5.23 \mathrm{e}-06$ & -4.31 & 0.000 & $-0.0000331-0.000012$ \\
& Constant & -0.5072473 & 0.5434615 & -0.93 & 0.356 & -1.6027020 .5878474 \\
\hline
\end{tabular}

Pseudo R-square $=0.86$, Number of obs $=48$, Prob $>$ F $=0.001$, AIC $=88.64059$. 


$$
\begin{gathered}
Y_{t}=-0.507-0.0000259 Z_{0 t}+0.0000731 Z_{1 t} \\
-0.0000225 Z_{2 t}
\end{gathered}
$$

Although at the $75^{\text {th }}$ percentile from Table 5 , all the parameter estimates of the $1^{\text {st }}, 2^{\text {nd }}$, and the $3^{\text {rd }}$ lags are significant except the constant with R-square of $86 \%$ and AIC $=88.64$. Hence, we proceeded to Table 6 .

From Table 6, we observed that all the parameter estimates are significant at the $95^{\text {th }}$ percentile. Also the R-square value is high, that is about $96 \%$ of the dependent variable can be explained by the independent variables with AIC $=101.84897$. The resulting transformed equation is given below:

$$
\begin{gathered}
Y_{t}=-1.303292-0.0000435 Z_{0 t}+0.0000941 Z_{1 t} \\
-0.0000257 Z_{2 t}
\end{gathered}
$$

Estimating the coefficients of the original variables, we have

$$
\begin{gathered}
\hat{\beta}_{0}=\hat{a}_{0}=-0.0000435 \\
\hat{\beta}_{1}=\hat{a}_{0}+\hat{a}_{1}+\hat{a}_{2} \\
=-0.0000435+0.0000941 \\
-0.0000257=0.0000231 \\
\hat{\beta}_{2}=\hat{a}_{0}+2 \hat{a}_{1}+4 \hat{a}_{2} \\
=-0.0000435+(2 * 0.0000941) \\
-(4 * 0.0000257)=0.000042 \\
\hat{\beta}_{3}=\hat{a}_{0}+3 \hat{a}_{1}+9 \hat{a}_{2} \\
=-0.0000435+(3 * 0.0000941) \\
-(9 * 0.0000257)=0.000008
\end{gathered}
$$

Therefore, we have the QPDL estimated model as: $Y_{t}=-1.303292-0.0000435 X_{0}+0.0000231 X_{t-1}$ $+0.000042 X_{t-2}+0.000008 X_{t-3}$

\subsection{Test of the Parameter Estimates.}

Khmaladze [22] tests whether the location-shift of the three QPDL lag coefficients are constant with respect to tau. The function Khmaladze Test computes both a joint test that all the covariate effects satisfy the null hypothesis (Tn), and a coefficient by coefficient version of the test.

$$
\mathrm{Tn}=4.314855
$$

We fail to reject the joint test statistic of the hypothesis that all the slope parameters of the model satisfy the hypothesis at critical values found in Ref. $[17,23]$ which are:

$1 \%$ critical value 5.350 and $5 \%$ critical value 4.523 with $\mathrm{x} 1=$ proz0 parameter estimate, $\mathrm{x} 2=$ proz1 and $\mathrm{x} 3$ $=$ proz2. The test statistic estimates for $\mathrm{x} 1$ to $\mathrm{x} 3$ are given below:

$$
\mathrm{THn}=\mathrm{x} 1 \mathrm{x} 2 \mathrm{x} 3
$$

\subsection{1 .3466831 .724839}

Hence the individual slope parameters satisfy the null hypothesis.

\subsection{Analysis of Variance}

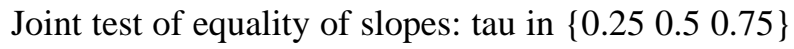
is summarised in Table 7.

From the analysis of variance, we reject that there is no differences between the slopes of all the tau values at $5 \%$ significance level.

The AIC's for the QPDL rubber estimates were 54.81562, 66.28824, 88.64059 and 101.849 at tau $=$

Table 6 Conditional quantile polynomial distributed lag (QPDL) 0.95 quantile regression parameter estimates, standard errors and confidence intervals for production and price of rubber.

\begin{tabular}{lllllll}
\hline Quantile & Price & Coef & Std. Err. & $\mathrm{t}$ & $\mathrm{P}>|\mathrm{t}|$ & [95\% Conf. Interval] \\
\hline 0.95 & Proz0 & -0.0000435 & $4.91 \mathrm{e}-06$ & -8.86 & 0.000 & $-0.0000534-0.0000336$ \\
& Proz1 & 0.0000941 & $5.02 \mathrm{e}-06$ & 18.75 & 0.000 & 0.0000840 .0001043 \\
& Proz2 & -0.0000257 & $1.17 \mathrm{e}-06$ & -21.97 & 0.000 & $-0.0000281-0.0000234$ \\
& Constant & -1.303292 & 0.4239428 & -3.07 & 0.004 & $-2.157693-0.4488914$ \\
\hline
\end{tabular}

Pseudo R-square $=0.96$, Number of obs $=48$, Prob $>$ F $=0.0031$, AIC $=101.84897$.

Table 7 Quantile regression analysis of variance table.

\begin{tabular}{llll}
\hline Df & ResidDf & F value & $\operatorname{Pr}(>\mathrm{F})$ \\
\hline 6 & 138 & 2.3588 & 0.0336 \\
\hline
\end{tabular}


(Intercept)

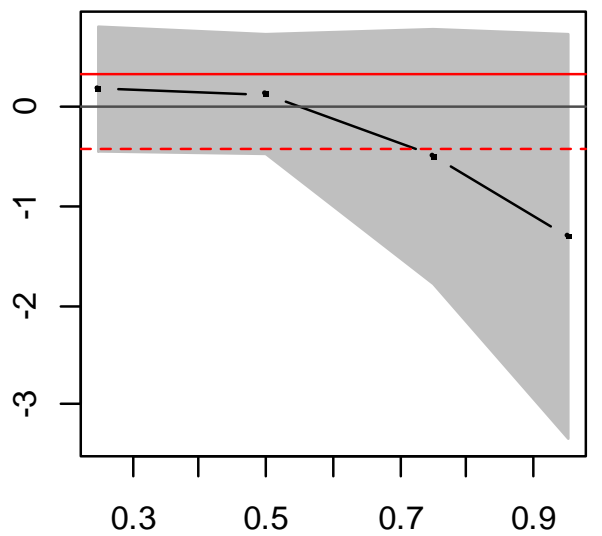

proz1

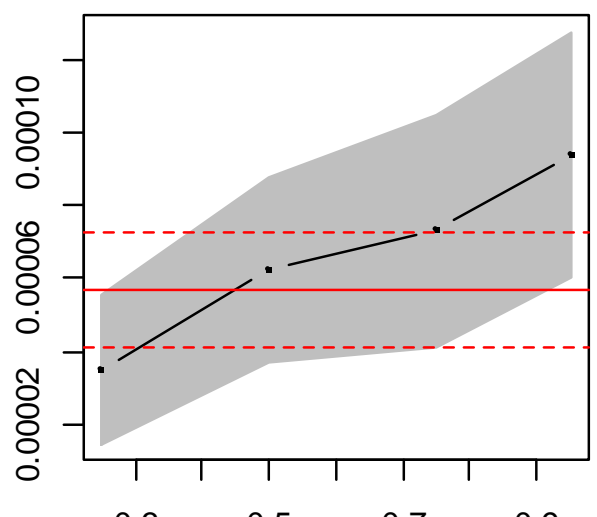

prozo

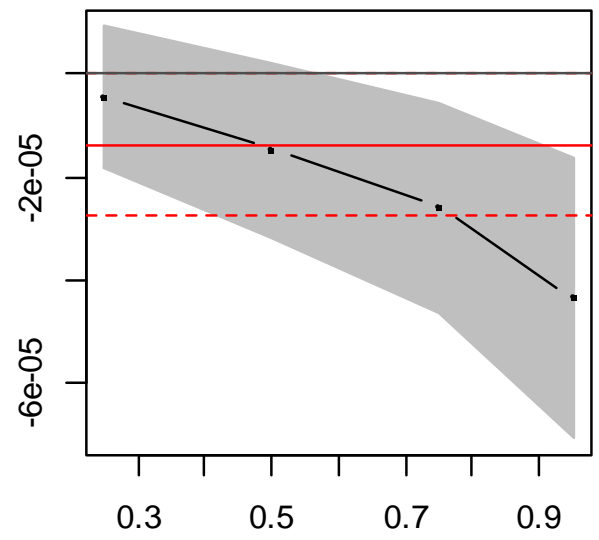

proz2

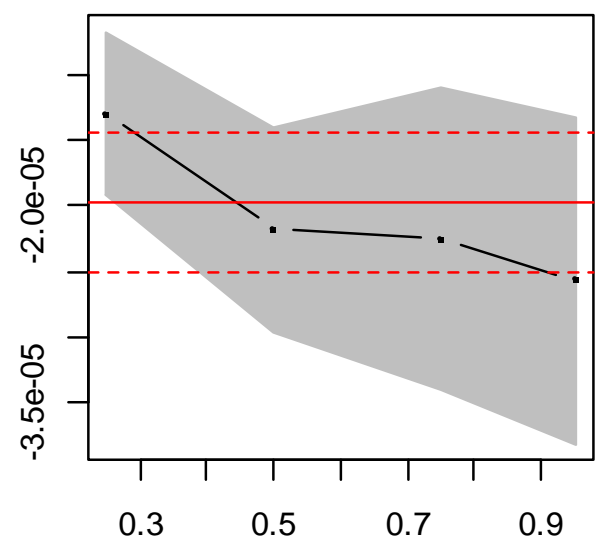

Fig. 1 Conditional quantile polynomial regression coefficient plotted as a function of tau $(\alpha)$ for the production and price of rubber. The solid black line indicates the quantile regression point estimates; the lighter grey region is a pointwise $95 \%$ confidence band.

0.25, 0.50, 0.75 and 0.95, respectively, and the PDL has $\mathrm{AIC}=75.79$ and $\mathrm{BIC}=85.148$. The AIC values for the QPDL at lower quantiles were smaller than the AIC for the PDL and higher than the PDL at higher quantilse.

\subsection{Graphical Interpretation}

Fig. 1 gives the QPDL parameter estimates of rubber production which enables us to compare and make inference, on the fitted model in the coefficient plots. The solid black line in these plots is the point estimate of the respective quantile regression fits, and the lighter grey region indicates a 95\% confidence region. The solid (horizontal) black line in some of the plots indicates a null effect. The solid straight horizontal line in each of the plots indicates the estimated OLS effects with a red dashed lines around it representing its 95\% confidence intervals.

From the intercept we could observe that quantile estimates differ significantly from the OLS and lies below the OLS line, and also decreases along higher quantiles.

From the production lag (proz0), the estimates decreases across the quantiles, and differ significantly from the OLS since it lies far above, and below the 95\% confidence interval at both lower and higher quantiles.

From (proz1) there is an increase across the quantiles, and this differs significantly from the OLS since it lies below the OLS at lower quantile and above it at higher quantiles. 
Although (proz2) decreases across the quantiles, it is not significantly different from the OLS estimates since it lies within the $95 \%$ confidence interval of the OLS estimates.

\section{Conclusions}

The hypothesis that there are no differences between the estimated slopes of all the tau values at $5 \%$ Significance level was rejected. Thus, there is a significant difference between all the slopes. The AIC's for the QPDL rubber estimates were 54.81562, 66.28824, 88.64059 and 101.849 at tau $=0.25,0.50$, 0.75 and 0.95 , respectively, and the PDL has AIC = 75.79 and $\mathrm{BIC}=85.148$.

Hence, we observe that there is an impact of both current production and three past productions on the pricing of rubber as suggested by lag effects in economic theory.

From the above analysis it could be observed that the QPDL models are better estimators than the PDL models, since the R-square measures of the QPDL models are higher than that of the PDL models and also the parameter estimates of the QPDL models are significant unlike that of the PDL models which were not significant.

We also observed that the "Ordinary" regression provides only a single summary measure for the conditional distribution of response (predictand) - the mean - given the predictor whereas quantile regression provides a more complete picture of the conditional distribution.

This idea naturally lends itself to all the generalizations that have followed ordinary regression. Many problems would actually benefit more from the prediction of extreme values rather than the mean.

From the graphical presentation, the true differences could be observed.

\section{References}

[1] Tomek, W. G., and Robinson K. L. 2003. Agricultural Product Prices. Cornell University Press.

[2] Yilmaz, Demircan, H. V., and Erel, G. 2006. "The
Comparative Analysis of Production Cost and Revenue in Potato Growing in Some Important Province Producing Potato.” Journal of the Agricultural Faculty of Suleyman Demirel University 1: 22-32.

[3] Liu, D. J., Keiser, H. M., Mount, T. D., and Forker, O. D. 1991. "Modelling the U.S Dairy Sector with Government Interventions.” West Journal of Ag. Econ. 16: 360-373.

[4] Koyck, L. 1954. Distributed Lags and Investment Analysis. North-Holland Publishing, Co.

[5] Nerlov, M., and Peter Jr., R. K. 1984. "The Supply Response of Rubber in Sri Lanka.” World bank Staff Working Paper 657.

[6] Nerlove, M. 1958. The Dynamic of Supply: Estimation of Farmer's Response to Price. John Hopkins University Press.

[7] Pindyck, R., and Rubinfield, D. 1981. Econometric Models and Forecasts. New York: McGraw Hill, Inc.

[8] Muth, J. F. 1961. "Rational Expectation and the Theory of Price Movements.” Econometrica 29.

[9] Almon, S. 1965. "The Distributed Lag Between Capital Appropriations and Expenditure.” Econometrica 33: 178-196

[10] Schmidt, P. 1974. "A Modification of the Almon Distributed Lag.” Journal of the American Statistical Association 69: 679-681.

[11] Bassett, G., and Koenker, R. 1982. "Tests of Linear Hypotheses and L1 Estimation.” Econometrica 50: 1577-1583.

[12] Gutenbrunner, C., Jureckova, J., Koenker, R, and Portnoy, S. 1993. "Tests of Linear Hypotheses Based on Regression Rank Scores.” J. of Nonparametric Statistics 2: 307-331.

[13] GalvaoJr, A.F., Montes-Rojas, G. and Park, S.Y. (2013). "Quantile Autoregressive Distributed Lag Model with an Application to House Price Returns”. Oxford Bulletin of Economics and Statistics, 75(2), 307-321

[14] Nyblom, J. 1989. "Testing For The Constancy Of Parameters Over Time.”Journal of the American Statistical Association 84: 223-230.

[15] Koenker, R. W. 2005. Quantile Regression. Cambridge U. Press.

[16] Chaudhuri, P. 1991. "Nonparametric Estimates of Regression QuantilesAnd Their Local Bahadur Representation.”Annals Of Statistics 19: 760-777.

[17] Koenker, R., and Xiao, Z. J. 2002. "Inference on the Quantile Regression Process.”Econometrica 81: 1583-1612.

[18] FAOSTAT, “Sri Lanka Annual Data (1966-2010)”, Accessed February 10, 2014. http://faostat.fao.org.

[19] World Bank Pink Sheet “Annual Data (1961-2013)”. Accessed August 08, 2014. http://econ.worldbank.org. 

Application to Rubber Price Returns

[20] Chen, K., Ying, Z., Zhang, H., and Zhao, L. 2008.“Analysis of Least Absolute Deviations.”Biometrika 95: 107-122.

[21] Koenker, R. W., and Bassett, G. W. 1982. "Robust Tests for Heteroscedasticity based on Regression Quantiles.”Econometrica 50: 43-61.
[22] Khmaladze, E. 1981. "Martingale Approach in the Theory of Goodness-of-fit Tests," Theory of Prob. and its Apps 26: 240-257.

[23] Qu, Z. 2008. "Testing For Structural Change In Regression Quantiles.”Journal Of Econometrics 146: 170-184. 